\title{
Signet ring cell-type adenocarcinoma arising in a mature teratoma of the testis
}

\author{
HONG KOO HA, WAN LEE, SANG DON LEE, JEONG ZOO LEE and MOON KEE CHUNG
}

Department of Urology, Pusan National University School of Medicine, Busan 602739, Korea

Received October 23, 2009; Accepted December 29, 2009

DOI: 10.3892/ol_00000053

\begin{abstract}
A 48-year-old male who presented with an enlarged right scrotum was diagnosed with malignant transformation of testicular teratoma. Physical examination revealed a right scrotal mass of hard consistency with no inguinal lymphadenopathy. Since prepuberty, his right testis had been larger than the left one, with no pain or tenderness. Computed tomography and bone scan revealed retroperitoneal lymphadenopathy and multiple bone metastases. Right orchiectomy was performed immediately, and a pathological examination revealed a mature teratoma associated with adenocarcinoma, showing signet ring cell differentiation. Cisplatin-based combination chemotherapy was administered; however, the metastatic lesions progressed, and the patient succumbed to the disease after 15 months. Only a few cases of primary malignant transformation of teratoma in the testis have been reported, and this is the first case report of primary malignant transformation of teratoma in the testis with signet ring cell-type differentiation.
\end{abstract}

\section{Introduction}

Malignant transformation of teratoma (MTT) is the transformation of a somatic teratomatous component of a germ cell tumor to an aggressive non-germ cell tumor phenotype (1). There have been many reports of secondary MTT after chemotherapy or radiotherapy in metastatic lesions and primary MTT in the ovary; however, primary MTT of the testis without chemotherapy or radiotherapy has rarely been reported. Due to the rarity of this entity, clinical features and prognosis have not yet been identified. However, three cases of MTT of the testis have been reported; one revealed colon type adenocarcinoma, and the other two did not describe the specific histology. Therefore, this is the first case report of MTT of the testis with gastric adenocarcinoma differentiation. We were presented with a patient with MTT of the testis

Correspondence to: Dr Hong koo Ha, Department of Urology, Pusan National University Hospital, 305 Gudeok-Ro, Seo-Ku, Busan 602739, Korea

E-mail: hongkooha@naver.com; hongkooha@pusan.ac.kr

Key words: adenocarcinoma, testis, teratoma associated with signet ring cell-type adenocarcinoma who had a symptomless testicular mass for several decades.

\section{Case report}

A 48-year-old male visited our facility, presenting with a large, intermittent painful mass in his right scrotum. The fistsized mass showed a firm consistency, but without inguinal lymphadenopathy. The patient reported that the right testis had been slightly larger than the left one since childhood, but there were no symptoms. The mass had been growing very slowly without pain until recently when the patient experienced intermittent pain in the right scrotum.

We received the ethics committee's approval and the patient's written informed consent. Ultrasonography showed a right hydrocele with the heterogeneous mass. Computed tomography (CT) revealed a right testicular mass measuring $5 \mathrm{~cm}$ and multiple aortocaval and paraaortic lymphadenopathies (Fig. 1). A bone scan showed increased radioisotope uptake at the 2nd and 4th lumbar vertebrae, sternum, right scapula and ribs (Fig. 2).

Serum $\alpha$-fetoprotein, $\beta$-human chorionic gonadotropin, carcinoembrionic antigen and other laboratory results were within normal limits. Radiologic and clinical evaluations found no other primary malignant tumors.

Following diagnosis of a primary testicular tumor with multiple metastases, right orchiectomy with high inguinal incision was performed. Pathologically, the tumor measured $10.5 \times 8.3 \times 7.0 \mathrm{~cm}$ and was classified as a mature teratoma associated with adenocarcinoma showing signet ring celltype adenocarcinoma differentiation and presenting invasion into the spermatic cord with involvement of the epididymis (Fig. 3). There was perineural invasion, but no evidence of lymphovascular tumor emboli was found. The spermatic cord resection and specimen margins were free of tumors. Results from immunohistochemical staining showed that tumor cells were positive to CDX-2, CK20 and focal-positive to CK7, but negative to TTF-1.

Positron emission tomography showed an increased uptake at the sternum, lumbar vertebrae, rib and retroperitoneal lymph node, with no abnormal uptake in the gastrointestinal tract (Fig. 4). The patient complained of persistent back pain, and a thoracolumbar spinal CT showed an osteolytic lesion extending into the paravertebral area at the 2 nd and 4th lumbar vertebral bodies (Fig. 5). Biopsy of the oeteolytic 


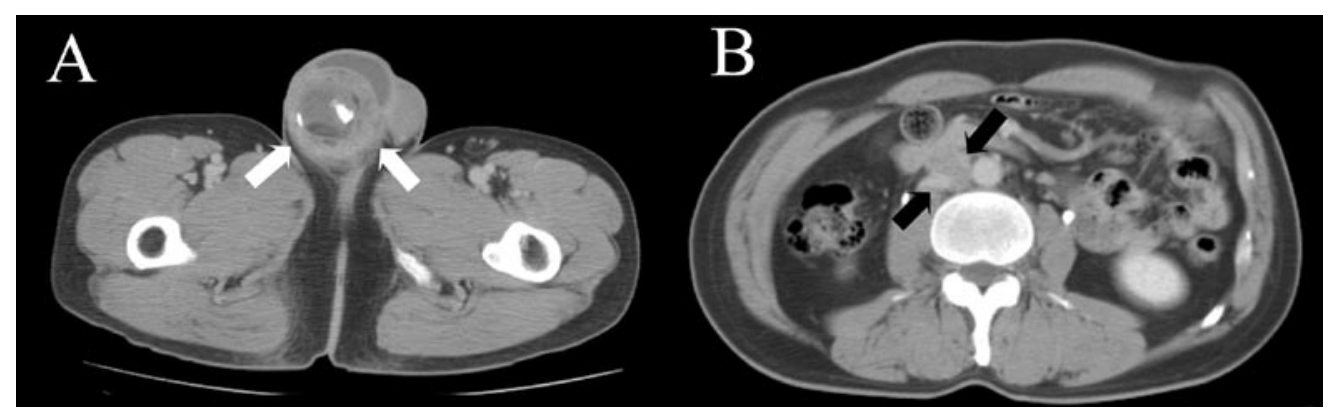

Figure 1. Enhanced pelvic CT shows a $5.0 \times 5.0 \mathrm{~cm}$ mass in the right testis (white arrows). The tumor is round with a thick wall and is irregularly enhanced in contrast to the low-density internal area, suggesting fat and focal calcifications. The tumor is accompanied by a mild hydrocele (A). An enlarged retroperitoneal lymph node $(1.7 \mathrm{~cm})$ is shown in the aortocaval area (black arrows) (B).

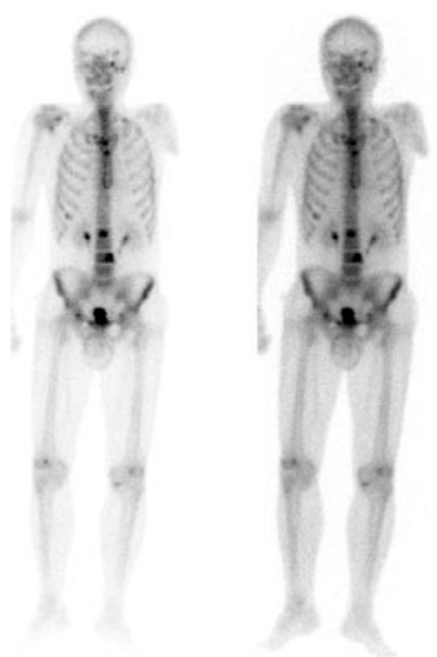

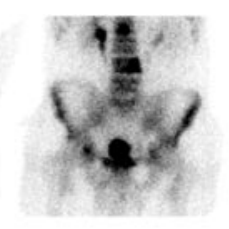

ANTERIOR

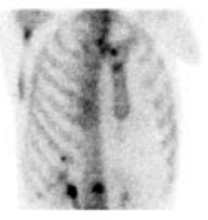

LPO

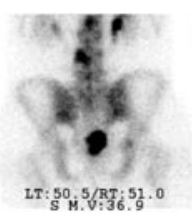

POSTERIOR_SS

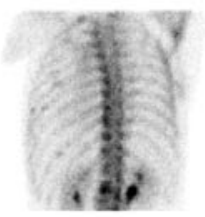

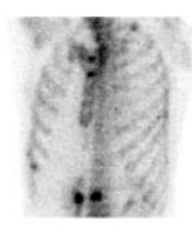

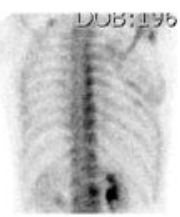

LAO
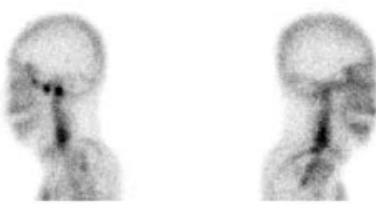

L. LAT
RPO

R. LAT

Figure 2. Bone scan shows areas of multiple metastases, including the 2nd and 4th lumbar vertebrae, sternum, right scapula and ribs.

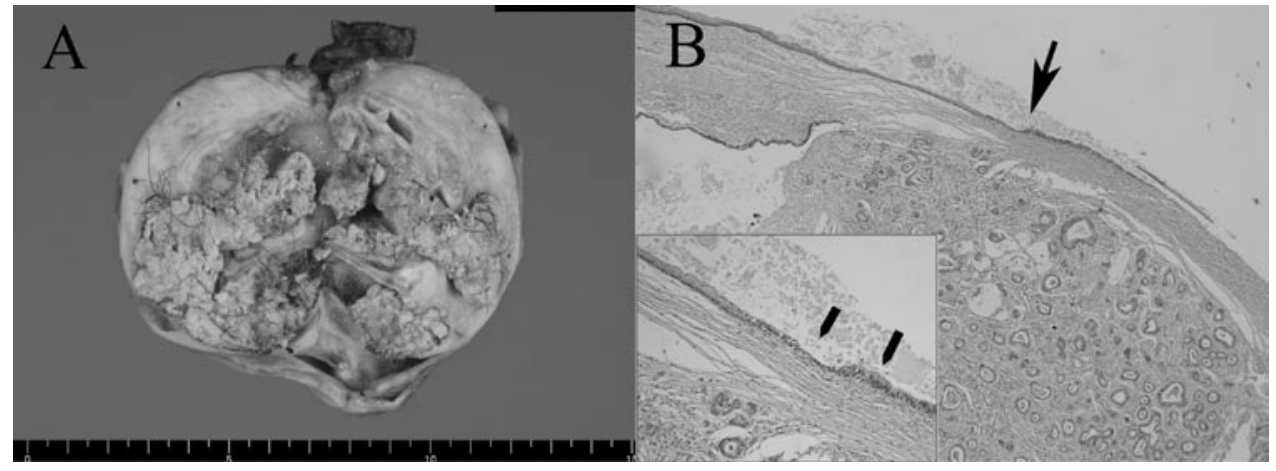

Figure 3. Mature teratoma associated with adenocarcinoma showing intestinal differentiation. (A) Gross appearance of the tumor. Nearly the whole testis is replaced by tumor mass and fibrous tissue. The cut surface of the mass shows yellowish, white grumous material, occasional mucoid material and some hair. (B) Microscopic findings of the tumor. The squamous epithelium is converted to columnar cells (arrow). Abundant glandular structures are noted beneath the epithelium. Tumor cells show irregularity of glandular structure, and some cells reveal a scattered pattern. The nucleolus is dense and displaced by cytoplasm, an indication of signet ring type adenocarcinoma (H\&E staining).

lesion at the 4th lumbar vertebral body revealed metastatic adenocarcinoma upon pathology. A 5-FU- and cisplatinbased combination of chemotherapy and radiotherapy was administered. However, a follow-up bone scan and CT showed that the metastatic lesions had progressed, and the patient succumbed to the disease 15 months later.

\section{Discussion}

Teratomas are the most common testicular tumors found in prepubertal children (2). Prepubertal mature teratoma shows a benign clinical course; however, teratoma in adults has a tendency to metastasize (3). In primary testicular tumors, tera- 

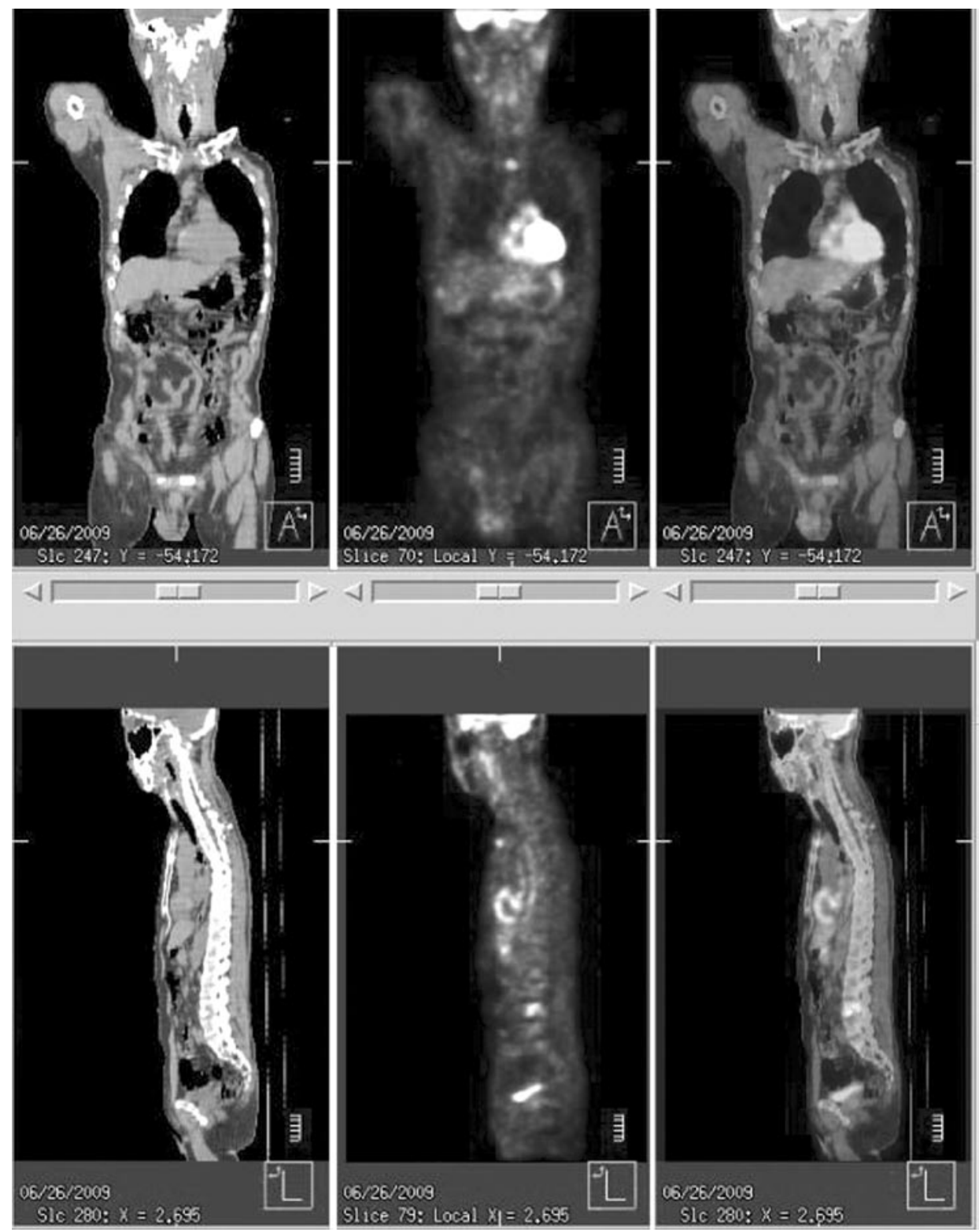

Figure 4. Whole body PET/CT shows hypermetabolic lesions with fluorodeoxyglucose uptake at the sternum, lumbar vertebrae, rib and aortocaval lymph node, with no abnormal uptake in the stomach and intestine.

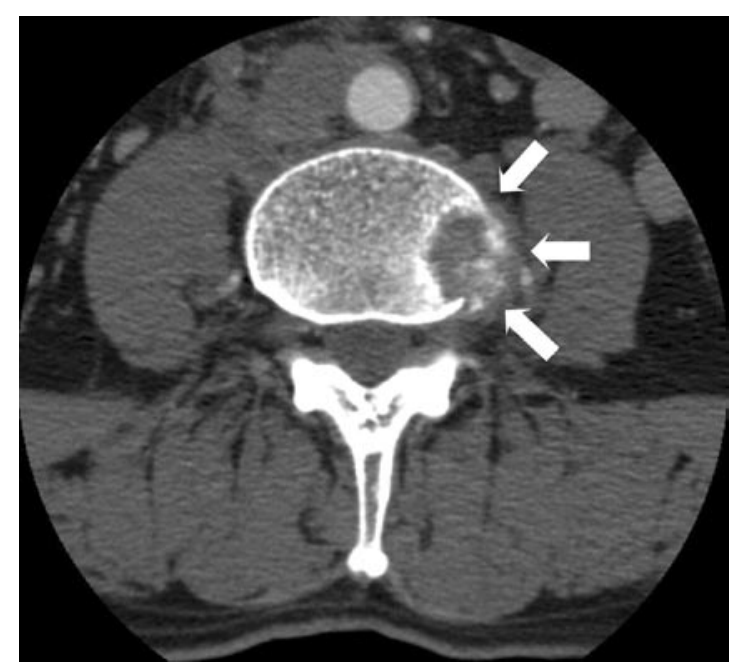

Figure 5. Thoraco-lumbar CT shows an osteolytic lesion extending into the paravertebral area on the left side of the 4th lumbar vertebrae. toma rarely undergoes transformation into a somatic malignant tumor. MTT is used to describe a non-germ cell tumor arising in the teratoma. MTT of ovarian cystic teratomas has been well documented, with an incidence of approximately $2 \%$ (4). However, MTT arising in mature teratomas in extraovarian sites is rare (5). Subsequently, a review of the literature found only three case reports of MTT in primary testicular teratoma. One was a case of MTT of the testis with colonic differentiation, while the other two cases did not describe the specific pathology. Michal et al (6) first reported on a primary signet ring stromal tumor of the testis; however, this case was not related to MTT. Therefore, this is the first case report on MTT of the testis with signet ring adenocarcinoma differentiation.

The mechanism of MTT in the testis remains poorly understood. Two mechanisms for the development of MTT have been postulated: malignant differentiation of the totipotential embryonal carcinoma cell to a neoplasm of somatic phenotype, or malignant transformation of mature teratoma 
elements (7). Koseoglu et al (5) also suggested the following clinical mechanisms of MTT in the testis: i) chemotherapy- or radiotherapy-induced MTT and ii) de novo MTT. Mediastinal, retroperitoneal and metastatic MTT have typically been associated with chemotherapy or radiotherapy. Mediastinal or retroperitoneal mature teratomas are sensitive to the transforming effect of chemotherapy or radiotherapy $(8,9)$. Teratomas in these regions are usually transformed into the sarcomatous type as a result of chemotherapy or radiotherapy. In addition, malignant transformation of metastatic mature teratomas may frequently occur as a result of the same treatment. Some authors have reported on the diagnosis of MTT of the testis with malignant transformation of a pre-existing teratoma (5). However, these results involved patients with MTT of the testis who underwent chemotherapy or radiotherapy; few cases of MTT in a primary testicular tumor with no previous treatment have been reported. In addition, MTT associated with adenocarcinoma in retroperitoneum, mediastinum, or metastatic teratomas is rare. Moreover, primary mature teratoma with malignant transformation associated with an adenocarcinoma phenotype is extremely rare. Therefore, the mechanism of primary testicular MTT remains unknown.

Immunohistochemical study is a useful tool for the identification of the exact type of adenocarcinoma. Park et al (10) found an expression of CDX-2 in $60.9 \%$ of stomach adenocarcinoma patients and also suggested the value of determining tissue-specific immunohistochemical stains in the diagnostic differentiation of adenocarcinomas. These authors reported that the positive predictive value of CDX-2(+), CK7(+), TTF-1(-) and CK20(-) in signet ring type adenocarcinoma was $85.7 \%$. In the present case, the specimen found in the testis showed the same immunohistochemical response as stomach adenocarcinoma. Kaseoglu et al (5) demonstrated that adenocarcinoma originating from colonic glands in the testis showed CEA(+), CA 19-9(+), CK20(+) and CK7(-) upon immunohistochemical staining results, which differed from this case.

Park et al (11) found that the incidence rates of MTT were $0.8 \%$ in all mature teratomas in the ovary, and carcinoma components were present in solid portions in cysts and thickened cystic walls in ovarian MTT. Kido et al (12) reported that malignant tumors arising in ovarian teratoma have a solid component region upon contrast enhancement, with transmural extension and irregular invasion through the septa to the adjacent organs. In our case, similar to the ovarian teratoma, contrast enhancement during the pathologic examination also identified adenocarcinoma in the solid portion of the cyst.
Kuo et al (13) reported an excellent prognosis in patients with signet ring stromal tumor of the testis. In addition, Asano et al (8) reported no recurrence after radical orchiectomy in patients with non-metastatic MTT of the testis. However, when metastasis occurs, prognosis of MTT of the testis is dependent on the histologic phenotype. MTT of the testis with adenocarcinoma usually requires an aggressive course of treatment. Kasai et al (9) reported on a patient who, despite cisplatin-based chemotherapy, succumbed to MTT at 8 months after orchiectomy. In the present case, the metastatic lesions also progressed despite cisplatin-based combination chemotherapy. Therefore, early detection of MTT is critical.

\section{References}

1. Comiter CV, Kibel AS, Richie JP, Nucci MR and Renshaw AA: Prognostic features of teratomas with malignant transformation: a clinicopathological study of 21 cases. J Urol 159: 859-863, 1998.

2. Pohl HG, Shukla AR, Metcalf PD, et al: Prepubertal testis tumors: actual prevalence rate of histological types. J Urol 172: 2370-2372, 2004.

3. Shukla AR, Woodard C, Carr MC, et al: Experience with testis sparing surgery for testicular teratoma. J Urol 171: 161-163, 2004.

4. Sumi T, Ishiko O, Maeda K, Haba T, Wakasa K and Ogita S: Adenocarcinoma arising from respiratory ciliated epithelium in mature ovarian cystic teratoma. Arch Gynecol Obstet 267: 107-109, 2002.

5. Koseoglu RD, Parlaktas BS, Filiz NO, Erdemir F, Uluocak N and Tulunay O: Adenocarcinoma originating from a mature teratoma of the testis. Kaohsiung J Med Sci 23: 265-268, 2007.

6. Michal M, Hes O and Kazakov DV: Primary signet-ring stromal tumor of the testis. Virchows Arch 447: 107-110, 2005.

7. El Mesbahi O, Terrier-Lacombe MJ, Rebischung C, Theodore C, Vanel D and Fizazi K: Chemotherapy in patients with teratoma with malignant transformation. Eur Urol 51: 1306-1312, 2007.

8. Asano T, Kawakami S, Okuno T, et al: Malignant transformation in a mature testicular teratoma left untreated for more than 50 years since childhood. Scand J Urol Nephrol 37: 177-178, 2003.

9. Kasai T, Moriyama K, Tsuji M, Uema K, Sakurai N and Fujii Y: Adenocarcinoma arising from a mature cystic teratoma of the testis. Int J Urol 10: 505-509, 2003.

10. Park SY, Kim BH, Kim JH, Lee S and Kang GH: Panels of immunohistochemical markers help determine primary sites of metastatic adenocarcinoma. Arch Pathol Lab Med 131: 1561-1567, 2007.

11. Park JY, Kim DY, Kim JH, Kim YM, Kim YT and Nam JH: Malignant transformation of mature cystic teratoma of the ovary: experience at a single institution. Eur J Obstet Gynecol Reprod Biol 141: 173-178, 2008.

12. Kido A, Togashi K, Konishi I, et al: Dermoid cysts of the ovary with malignant transformation: $\mathrm{Mr}$ appearance. AJR Am J Roentgenol 172: 445-449, 1999.

13. Kuo CY, Wen MC, Wang J and Jan YJ: Signet-ring stromal tumor of the testis: a case report and literature review. Hum Pathol 40: 584-587, 2009. 